\title{
ALMA observations of local analogs of high-redshift star-forming galaxies
}

\section{Thiago Gonçalves}

\author{
Valongo Observatory, UFRJ, Brazil
}

\begin{abstract}
I will present the result of two observational projects using ALMA to investigate the properties of the molecular gas in low-redshift $(z \sim 0.2)$ ultraviolet-luminous galaxies. These objects are extremely dense, highly star-forming and very metal-poor compared to other galaxies of similar stellar mass at the same redshifts, justifying their use as analogues to distant mainsequence galaxies in an attempt to understand the interplay between gas and star formation under similar conditions in the early universe. Firstly, we have observed the most metal-poor objects in our sample, in order to determine whether metallicity plays a role in CO emissivity of the molecular regions in these galaxies. Our four non-detections, with stringent upper limits, shows that $\mathrm{CO}$ is severely depleted, even under turbulent conditions. We have also observed one object with high spatial resolution, comparing data from $\mathrm{CO}$ emission and hydrogen recombination lines down to a resolution of $\sim 400 \mathrm{pc}$, allowing for a detailed analysis of the conversion of gas into new stars. We are able to compare star formation laws in individual clumps and the surrounding ISM, highlighting the difference between star formation efficiencies in each environment within the galaxy. Finally, the high-resolution data offers interesting insights on the growth of supermassive black holes in these galaxies: our combined multiwavelength data shows that there must be a low-mass $\left(10^{5} M_{\odot}\right)$ black hole in the center of the galaxy, while bolometric luminosity in the central region is dominated by star formation activity.
\end{abstract}

